\title{
CONTROLE DA SINCRONIZAÇÃO EM UM AGRUPAMENTO DE REDES NEURONAIS
}

\author{
Ewandson luiz lameu*, Antonio Marcos Batista*, Kelly Cristiane Iarosz*, Carlos \\ Adalberto Schineider Batista ${ }^{\dagger}$, Ricardo Luiz Viana ${ }^{\dagger}$, Sergio Roberto Lopes ${ }^{\dagger}$ \\ *Pós-Graduação em Ciências/Fúsica \\ Universidade Estadual de Ponta Grossa \\ Ponta Grossa, Paraná, Brasil \\ ${ }^{\dagger}$ Departamento de Física \\ Universidade Federal do Paraná \\ Curitiba, Paraná, Brasil
}

Emails: ewandson.ll@gmail.com, antoniomarcosbatista@gmail.com, kiarosz@gmail.com, batiscarlos@gmail.com, viana@fisica.ufpr.br, lopes@fisica.ufpr.br

\begin{abstract}
Functional brain networks are composed of cortical areas that are anatomically and functionally connected. Statistical analyses suggest that the brain structure can be described as a clustered network, in which each cluster is a scale-free network possessing highly connected hubs. We have built a clustered scale-free network inspired in the cat cortex structure so as to study their dynamical properties. We focus on the synchronization of bursting activity of the cortical areas and how it can be suppressed by means of neuron deactivation through suitably applied light pulses. We show that is possible to effectively suppress bursting synchronization by acting on a single hub, because it is highly connected and have a strong influence over the network.
\end{abstract}

Keywords- Neuronal Networks, synchronization, synchronization control.

Resumo - As redes funcionais do cérebro são compostas por áreas corticais que estão anatomicamente e funcionalmente conectadas. Análises estatísticas sugerem que a estrutura cerebral pode ser descrita através uma estrutura de rede de redes, onde cada rede apresenta uma topologia livre de escala com hubs densamente conectados. Construímos uma uma rede de redes livres de escala inspirada no cortex cerebral do gato para, assim, estudar suas propriedades dinâmicas. Focamos na sincronização dos disparos neurais das áreas corticais e em como suprimir esse efeito por meio da desativação de um neurônio através de pulsos de luz. Mostramos que é possível suprimir a sincronização dos disparos perturbando apenas um único hub, pois este é altamente conectado e, portanto, possui grande influencia sobre a rede.

Keywords- Redes Neurais, sincronização, controle da sincronização.

\section{Introdução}

Os recentes avanços tecnológicos tem possibilitado a análise da estrutura e dinâmica do sistema nervoso como, por exemplo, o comportamento de neurônios, suas interações, algumas desordens neurológicas e os mecanismos que estão por trás do seu funcionamento. Os neurônios cerebrais organizam-se em grupos ou unidades funcionais chamadas de áreas corticais (visual, auditiva, motora, etc) responsáveis por processar as informações recebidas.

Podemos aproximar a organização topológica e as ligações entre os neurônios com uma rede, onde os nós (ou sítios) são os neurônios e as arestas (ligações) são os axônios e dendritos. Alguns estudos sobre a conectividade entre regiões do córtex cerebral de macacos e gatos mostraram as seguintes características: as áreas corticais organizam-se em aglomerados (clusters) de neurônios (Scannell and Young, 1993), (Scannell et al., 1995), (Hilgetag et al., 2000); com uma grande densidade de conexões e a presença de sítios altamente conectados, denominados hubs (Zemanova et al., 2006). Outra característica importante observada relaciona-se à comunicação en- tre as áreas corticais, ou seja, as conexões ocorrem não somente entre áreas próximas, mas também entre áreas distantes do córtex e são mediadas principalmente por seus hubs (Zamora-López et al., 2009). Como resultado destas características, uma rede neural apresenta certa hierarquia em sua organização espacial. Estas informações remetem a um modelo teórico que deve simular as conexões cerebrais de mamíferos onde em um nível hierárquico mais baixo encontramos uma estrutura de clusters com uma conectividade do tipo lei de potência, ou seja, uma rede do tipo livre de escala (Barabási and Albert, 1999). Em um nível hierárquico maior temos que as conexões entre os clusters ocorrem principalmente por intermédio de seus hubs.

A dinâmica dos disparos neuronais é formada por variações abruptas do potencial de membrana da célula, ou seja, um pico de potencial. Esta dinâmica pode variar desde um único pico até uma repetida sequência de picos que ocorre periodicamente. A dinâmica periódica de picos pode ser descrita por diversos sistemas teóricos. Neste trabalho utilizaremos redes de mapas acoplados, que apresentam o espaço e o tempo discretos, enquanto que as variáveis de estado são con- 
tínuas. Relacionado à dinâmica de uma rede neuronal, diversas desordens neurológicas, como mal de Parkinson e epilepsia (Kumar et al., 1998), estão relacionados com a sincronização da dinâmica dos disparos neurais. Em virtude destes problemas decorrentes da sincronização em uma rede neural, torna-se necessário o estudo dos processos pelos quais isto ocorre. Nos últimos anos alguns modelos foram propostos para estudar a sincronização dos disparos neuronais formas de suprimir este efeito (Batista et al., 2012), (Lameu et al., 2012). Trabalhos recentes mostram que é possível controlar eficazmente os disparos de neurônios geneticamente modificados através de estimulação por luz (Boyden et al., 2005).

Este trabalho esá organizado da seguinte maneira: na Seção 2 estudaos a sicronização em um modelo de rede de redes livre de escala com neurônios de Rulkov, na Seção 3 lidamos com o controle aplicado. Nossas conclusões estão na última seção.

\section{Sincronização em um Aglomerado de Redes Livres de Escala}

Existem diversos modelos matemáticos que simulam a atividade neuronal, desde equações diferenciais até mapas com tempo discreto. Utilizamos o modelo fenomenológico proposto por Rulkov (Rulkov, 2001):

$$
\begin{gathered}
x_{n+1}=\frac{\alpha}{1+x_{n}^{2}}+y_{n} \\
y_{n+1}=y_{n}-\sigma x_{n}-\beta,
\end{gathered}
$$

onde $n$ é o tempo discreto, $x_{n}$ e $y_{n}$ são as variáveis rápida e lenta, respectivamente. O parâmetro $\alpha$ afeta diretamente o período dos disparos, sendo escolhido de forma a fazer com que o mapa apresente um comportamento caótico durante os disparos. Os parâmetros $\sigma$ e $\beta$ descrevem a escala de tempo da variável lenta. A diâmica dos disparos neuronais estão demonstradas na Fig.(1).

Consideramos que os neurônios não são idênticos, ou seja, possuindo parametros internos distintos, no nosso caso, cada neurõnio possui um $\alpha$ diferente. Em virtude dos neurônios serem diferentes, uma sincronização completa dos seus disparos torna-se impossível, no entanto, uma sincronização da fase dos disparos ainda pode ocorrer (Pikovsky et al., 2001). Para estudar a sincronização de fase nós introduzimos uma fase $\phi$ para a dinâmica caótica dos disparos.

Um disparo começa quando a variável lenta $y_{n}$ apresenta um máximo local em períodos bem definidos de tempo. A duração de um disparo caótico, $n_{k+1}-n_{k}$, depende da variável rápida $x_{n}$ e flutua em um padrão irregular conforme $x_{n}$ evolui caoticamente. Podemos definir uma fase que descreve a evolução temporal de cada disparo

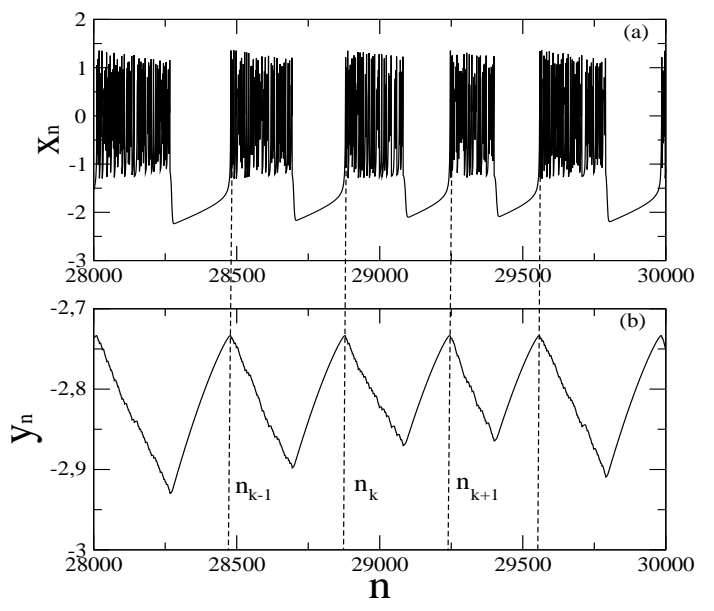

Figura 1: Evolução temporal da variável (a) rápida e (b) lenta do mapa de Rulkov, $\operatorname{com} \alpha=4,1$, $\beta$ e $\sigma=0,001$. Foram realizadas 30000 iterações, sendo 28000 descartadas.

variando de 0 a $2 \pi$ conforme $n$ evolui de $n_{k}$ para $n_{k+1}$ :

$$
\phi_{n}=2 k \pi+2 \pi \frac{n-n_{k}}{n_{k+1}-n_{k}},
$$

onde $k$ é um número inteiro e $n_{k}$ denota o instante em que ocorre o $k$-ésimo disparo. Um diagnóstico para sincronização pode ser dado pelo parâmetro de ordem de Kuramoto (Kuramoto, 1984) dado por:

$$
z_{n}=R_{n} \exp \left(\mathrm{i} \Phi_{\mathrm{n}}\right) \equiv \frac{1}{\mathrm{~N}} \sum_{\mathrm{j}=1}^{\mathrm{N}} \exp \left(\mathrm{i} \phi_{\mathrm{n}}^{(\mathrm{j})}\right)
$$

onde $R_{n}$ e $\Phi$ são a amplitude e o ângulo, respectivamente, de um vetor de fase unitário para uma rede unidimensional com condições de contorno periódicas. Se as fases dos neurônios estão descorrelacionadas, a suas contribuições no somatório da equação (4) será pequeno. No entanto, em um estado globalmente sincronizado a magnitude do parâmtro de ordem $R_{n}$ tende à unidade.

A média temporal da magnitude do parâmetro de ordem é dada por:

$$
\bar{R}=\frac{1}{T} \sum_{n=1}^{T} R_{n}
$$

quando os disparos entre os neurônios estão sincronizados temos $\bar{R} \approx 1$.

Construímos uma rede de redes formada por $M$ sub-redes cada uma apresentando uma topologia livre de escala. Redes livres de escala possuem poucos nós com muitas conexões (hubs) enquando que a maioria de seus nós possuem poucas conexões. As sub-redes foram conectadas entre si através de seus hubs, como demonstrado esquematicamente na Fig.(2). 


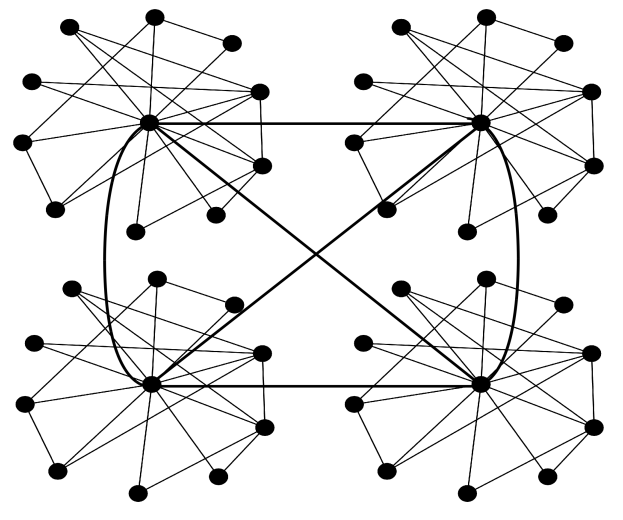

Figura 2: Rede formada por 4 sub-redes livres de escala idênticas acopladas globalmente por seus hubs.

A dinâmica dos disparos neuronais para esta rede é governado por:

$$
\begin{gathered}
x_{n+1}^{(i, l)}=\frac{\alpha^{(i, l)}}{1+\left(x_{n}^{(i, l)}\right)^{2}}+y_{n}^{(i, l)}+\mathcal{C}_{n}^{(i, l)} \\
y_{n+1}^{(i, l)}=y_{n}^{(i, l)}-\sigma x_{n}^{(i, l)}-\beta,
\end{gathered}
$$

onde $i=1,2, \ldots, N, l=1,2, \ldots, M, \sigma=\beta=0.001$, e osvalores de $\alpha^{(i, l)}$ foram uniformemente distribuidos no intervalo [4.1,4.4]. Consideramos o termo de acoplamento da seguinte forma:

$$
\mathcal{C}_{n}^{(i, l)}=\frac{\epsilon}{k^{(i, j)}} \sum_{l=1}^{N} g_{i l} x_{n}^{(l, j)},
$$

onde $j$ indica a sub-rede e $i$ um de seus sítios, $g_{i l}$ são o elementos da matriz conectividade $N \times N$, sendo que $g_{i l}=1$ se o sítio $i$ e $l$ estão conectados, e zero caso contrário. Podemos ver que o termo de acoplamento considera que o sítio $i$ sofre influência apenas dos $k^{(i, j)}$ sítios conectados a ele. Da mesma forma as M sub-redes estão globalmente acopladas entre si por mei de seus hubs com intensidade de acoplamento $\varepsilon_{h}$.

Analisamos a sincronização para um total de $M=10$ sub-redes, com $N=230$ neurônios cada. Na Fig.(3) fixamos o acoplamento entre os hubs em $\varepsilon_{h}=0,16$ e os dados nos mostram a média temporal do parâmetro de ordem de toda a rede (linha vermelha) e das sub-redes independentemente (linhas pretas) em função de $\varepsilon$. Como não ocorrerá sincronização total, pelo fato dos neurônios não serem idênticos, consideramos que o sistema está sincronizado quando $\bar{R} \geq 0,9$. No intervalo da região I a intensidade de acoplamento $\varepsilon$ não é suficiente para produzir sincronização na rede ou mesmo nas sub-redes, na região II as linhas pretas mostram as sub-redes sincronizadas de forma independente enquanto que a linha vermelha mostra que não há sincronização entre elas e, por fim, na região III todas a sub-redes estão sincronizadas entre si. Fica evidente destes resultados que a sincronização para $\varepsilon$ pequeno ocorre somente entre os elementos das sub-redes, e conforme ocorre o aumento de $\varepsilon$ as sub-redes sincronizam entre si.

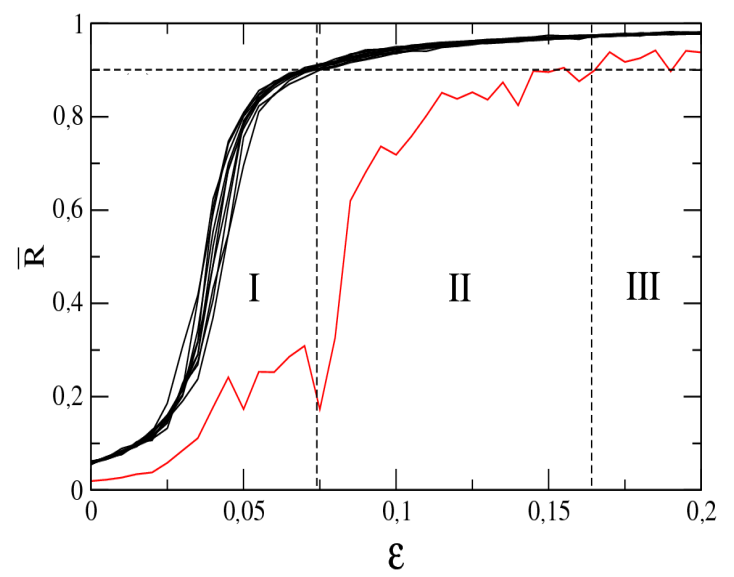

Figura 3: Consideramos $M=10, N=230$ e $\varepsilon_{h}=$ 0,16 . Média temporal do parâmetro de ordem em função da intensidade do acoplamento $\varepsilon$ para cada sub-rede (linhas pretas) e para toda a rede (linhas vermelhas).

\section{Supressão da Sincronização}

O controle das atividades neurais poderia revolucionar os tratamentos de desordens neurológicas. Para este fim, Boyden e colaboradores (Boyden et al., 2005) utilizaram duas proteínas fotossensíveis, Channelrhopisin-2 (ChR2) e a Halorhodopsin (Halo), em virtude dos efeitos que estas causavam na dinâmica dos disparos de neurônios in vitro quando expostas a luz com comprimento de onda específico. A proteína ChR2 quando exposta à luz na faixa do azul funciona como um canal iônico liberando cátions que atuam nos neurônios causando a ativação de disparos. Por outro lado, a proteína Halo, sensível à luz na faixa do amarelo, quando exposta funciona como uma bomba de íons cloro que provocam a interrupção das atividades neurais.

Com base nestas evidências buscamos suprimir a sincronia em domínios de acoplamento forte. Nosso método consiste em controlarmos a atividade de um dos hubs, que ligam as sub-redes entre si, de modo a simular uma perturbação provocada por foto-estimulação e verificar qual a influência desta na dinâmica e sincronização de toda rede. A perturbação baseia-se em inibir os disparos de um hub de acordo com uma distribuição de probabilidades de Poisson, ou seja, quando a perturbação é aplicada o $h u b$ sofre desligamentos aleatórios.

Para avaliar as consequências dessa influência na dinâmica de toda a rede, estudamos o com- 
portamento do parâmetro de ordem total em três regimes de aplicação: sem perturbação, com perturbação aplicada permanentemente e de forma intermitente, esta última consiste na alternância entre intervalos com perturbação e intervalos sem perturbação. Reforçando que nestes regimes a forma da perturbação é a mesma (desligamentos aleatórios), a única diferença está na maneira desta ser aplicada sobre o hub. A Fig. (4a) nos mostra a média do parâmetro de ordem total em função de $\varepsilon$, onde consideramos o sistema sincronizado para $\bar{R}$ acima de 0,9 . Vemos com o decréscimo do parâmetro de ordem total que a perturbação, tanto a aplicada de forma permanente quanto a intermintente, é capaz de evitar a sincronização total da rede. Portanto, não há a necessidade que esta seja aplicada constantemente sobre o sistema para evitar a sincronização.
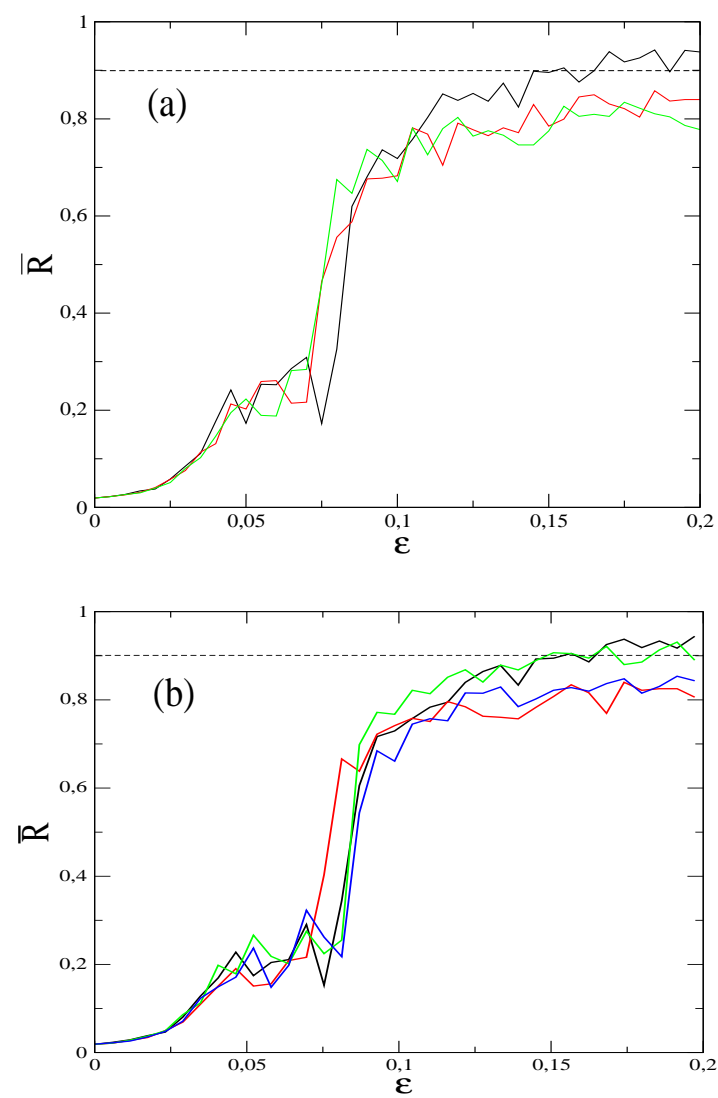

Figura 4: Média do parâmetro de ordem total $\bar{R}$ em função da intensidade de acoplamento $\varepsilon$, com $\varepsilon_{h}=0,16$. (a) Sistema sem perturbação (linha preta), com perturbação aplicada de forma permanente (linha vermelha) e com perturbação aplicada de forma intermitente (linha verde). (b) Sem perturbação (linha preta), com perturbação aplicada em 1 neurônio aleatório (linha verde), em 10 neurônios aleatórios (linha azul) e em um $h u b$ (linha vermelha). Foram feitas 100000 iterações sendo 50000 descartadas.
Analisamos, também, a perturbação sendo aplicada em diferentes neurônios na rede e comparamos os recultados com a perturbação qaundo aplicada em um hub. Vemos pela Fig.(4b) que para termos um efeito semelhante à perturbação sobre um $h u b$, devemos perturbar no mínimo 10 neurõnios aleatóriamente em uma sub-rede para suprimir a sincronização total do sistema.

\section{Conclusões}

Apresentamos neste trabalho um modelo de rede de redes onde a dinâmica de cada neurônio foi descrita pelo mapa de Rulkov, sendo que cada sub-rede do sistema apresentava uma topologia do tipo livre de escala. Demonstramos a possibilidade de ocorrer sincronização de fase dos disparos neuronais de toda a rede, sob domínio de acoplamento forte.

A aplicação de uma perturbação externa sobre um $h u b$, simulando uma foto-estimulação, demonstrou ser um método eficaz em suprimir os efeitos de sincronização total da rede. Essa perturbação pode ser aplicada de forma intermitente no tempo e quando aplicada sobre 10 neurônios aleatórios de uma sub-rede tem o mesmo efeito, em suprimir a sincronização, de quando aplicada sobre um hub.

\section{Agradecimentos}

A realização do trabalho foi possível devido ao auxílio financeiro das seguintes agências: CNPq, CAPES e Fundação Araucária.

\section{Referências}

Barabási, L. and Albert, R. (1999). Emergence of scaling in random networks.

Batista, C. A. S., Lameu, E. L., Batista, A. M., Lopes, S. R., Zamora-López, G., Kurths, J. and Viana, R. L. (2012). Phase synchronization of bursting neurons in clustered smallworld networks.

Boyden, E. S., Zhang, F., Bamberg, E., Nagel, G. and Deisseroth, K. (2005). Millisecondtimescale, genetically targeted optical control of neural activity.

Hilgetag, C. C., Burns, G. A., O’Neil, M. A., Scannell, J. W. and Young, M. P. (2000). Anatomical connectivity defines de organization of clusters of cortical areas in the macaque monkey an the cat.

Kumar, R., Lozano, A. M., Kim, Y. J., Hutchison, W. D. and Sime, E. (1998). Double-blind evaluation of subthalamic nucleus deep brain stimulation in advanced parkinson's disease. 
Kuramoto, Y. (1984). Chemical oscillations, waves and turbulence, Spring-Verlag.

Lameu, E. L., Batista, C. A. S., Batista, A. M., Iarosz, K. C., Viana, R. L., Lopes, S. R. and Kurths, J. (2012). Suppression of bursting synchronization in clustered scale-free (richclub) neuronal networks.

Pikovsky, A. S., Rosenblum, M. G. and Kurths, J. (2001). Synchronization: A Universal Concept in Nonlinear Sciences, Cambridge Uni-versity Press. DOI: 10.1017/CBO9780511755743

Rulkov, N. F. (2001). Regularization of synchronized chaotic bursts.

Scannell, J. W., Blakemore, C. and Young, M. P. (1995). Analysis of connectivity in the cat cerebral cortex.

Scannell, J. W. and Young, M. P. (1993). The connectional organization of neuronal systems in the cat cerebral cortex.

Zamora-López, G., Zhou, C. S. and Kurths, J. (2009). Graph analysis of cortical networks reveals complex anatomical communication substrade.

Zemanova, L., Zhou, C. S. and Kurths, J. (2006). Structural and functional clusters of complex brain networks. 